\title{
Emotion, Language, and the Brain
}

Sonja A. Kotz ${ }^{1 *}$ and Silke Paulmann ${ }^{2}$

${ }^{1}$ Max Planck Institute for Human Cognitive and Brain Sciences, Independent Research Group Neurocognition of Rhythm in Communication and ${ }^{2}$ Department of Psychology, University of Essex

\begin{abstract}
In social interactions, humans can express how they feel in what (verbal) they say and how (nonverbal) they say it. Although decoding of vocal emotion expressions occurs rapidly, accumulating electrophysiological evidence suggests that this process is multilayered and involves temporally and functionally distinct processing steps. Neuroimaging and lesion data confirm that these processing steps, which support emotional speech and language comprehension, are anchored in a functionally differentiated brain network. The present review on emotional speech and language processing discusses concepts and empirical clinical and neuroscientific evidence on the basis of behavioral, event-related brain potential, and functional magnetic resonance imaging data. These data allow shaping our understanding of how we communicate emotions to others through speech and language. It leads to a multistep processing model of vocal and visual emotion expressions.
\end{abstract}

\section{Introduction}

Research on emotional speech and language has recently regained immense popularity in the neuroscientific community. Overall, neuroimaging and clinical evidence confirms that communicating emotions is a highly complex and multidimensional phenomenon as we can use different modes or so-called channels of expression to communicate emotions. In the case of emotional speech verbal (i.e. lexical-semantics) and non-verbal (e.g. prosodic ${ }^{1}$ ) information sources are rapidly integrated. It is well accepted that this complex form of communication is mediated by a differentiated brain network (e.g. Schirmer and Kotz 2006; Wildgruber et al. 2006, 2009). However, empirical results do not always confirm engagement of all postulated brain regions in the network. Lack of activation in brain areas constituting an emotional speech network is often explained by different theoretical accounts of emotional speech, but may simply result from experimental constraints such as task demands, stimulus quality, -type, or -complexity (Ethofer et al. 2009a,b; Kotz et al. 2006). In addition, emotional word and sentence processing has not only been investigated in the auditory, but also in the visual modality (e.g. Harciarek et al. 2006; Kanske and Kotz 2007; Kissler et al. 2006; Landis 2006) adding to the complexity and the differentiation of a neural network supporting emotional expressions.

In the following, we will review past and recent clinical and empirical neuroimaging emotional speech and language evidence by zooming into the word and the sentence level in both the auditory and visual modality. While visual evidence will primarily provide insight into the processing of emotional semantics, data from the auditory domain will reveal the specific contributions of emotional prosody and semantics as well as their interaction in emotional speech. Consequently, this limits the scope of the paper to the neural correlates of emotional verbal (semantic) expressions and the integration of emotional verbal and non-verbal (prosodic) expressions. At the end of the review, we will 
provide a summary of major findings in the field and how they can be described by our recent model (Schirmer and Kotz 2006). We will also provide a short outlook on how this model can be extended by adding evidence from the visual modality which extends the growing work on the integration of verbal and non-verbal (i.e. facial and prosodic) emotional expressions already discussed elsewhere (e.g. Kreifelts et al. 2007, 2009; Wildgruber et al. 2009; Zupan et al. 2009).

\section{Emotional Speech and Language - What are the Critical Research Questions?}

One of the immanent early questions in emotion research was how specific emotional information is anchored in the brain leading to investigations of lateralization of function. This is not at all surprising as speech and language are considered to be primarily left hemisphere functions (e.g. Friederici 2002; Scott and Johnsrude 2003) while emotion processing has mainly been tied to the right hemisphere (e.g. Adolphs et al. 1996; Borod et al. 1998a,b). Due to the advancement of imaging techniques, such as positron emission tomography (PET), functional magnetic resonance imaging (fMRI), and event-related brain potentials (ERPs) as well as more sophisticated lesion studies, it has become evident that such an asymmetry of brain function(s) is relative rather than absolute. For instance, it has been shown that speech- and language-related processes recruit both hemispheres dependent on the type of information that is processed. Specifically, it has been suggested that segmental (distinct units or segments of speech, for instance, individual sounds or phonemes), lexical (information stored in the lexicon, for example, meaning), and syntactic (grammatical structure) information is processed in the left hemisphere, while suprasegmental (stretches over different segmental units) information is processed in the right hemisphere (e.g. Friederici and Alter 2004). Similarly, recent emotion research has identified a brain network that recruits both left and right hemisphere structures dependent on what kind of emotional information is processed (e.g. Adolphs et al. 2002; Davidson 2000). Thus, research has moved from the lateralization of function to more specific and sophisticated questions about how the brain responds to specific facets of emotion expressions, and how it constitutes respective brain network dynamics. Moreover, building on the observation that emotional speech and language is a multistep process (e.g. Schirmer and Kotz 2006; Wildgruber et al. 2006, 2009), it has become rather relevant to understand which brain areas are critically tied to specific processing steps. For example, we have argued that the processing of emotional speech can be subdivided into at least three processes: (1) sensory analysis, (2) derivation of emotional significance based on sensory cues, and (3) evaluation processes. Each of these subprocesses relies on different brain areas in both hemispheres. Toward the end of this review, we will suggest that the equivocal evidence with regard to the contributions of left and right hemispheres to emotional speech and language processing can be better understood if we assume that (1) lateralization may vary as a function of the proposed subprocesses, and that (2) lateralization can be substantiated by a brain network supporting these subprocesses beyond the right hemisphere. Recent empirical clinical, electrophysiological, and neuroimaging evidence will be addressed in the following to substantiate this claim.

\section{Which Brain Areas Constitute an Emotional Speech and Language Network? Answers from Clinical and Neuroimaging Studies}

Early clinical studies have contributed tremendously to our current understanding of the neural correlates of emotional speech and language. Various test batteries and paradigms 
have been applied (see Borod et al. 2000 for a summary of neuropsychological assessment tools). As will become apparent in the current review, some of the more popular tasks include identifying emotional categories in a forced-choice task from stimuli such as sentences, words, pseudowords, or syllables, discriminating between emotional speech stimuli (positive vs. negative, happy vs. pleasant surprise, etc.), and detecting mismatches between emotion information sources (e.g. semantics and prosody). Initially, clinical lesion studies aimed to specify respective hemispheric contributions to emotional speech and language. As early as 1874, John Hughlings Jackson reported that aphasic patients with lesions in the left hemisphere could still swear or produce emotional words (Jackson 1874; but see also Smith 1966) pointing to the involvement of the right, unaffected hemisphere in the production of emotion expressions. Later work by Heilman et al. (1975) reported that lesions in the right hemisphere tend to also disrupt emotional speech comprehension, while split-brain patients display verbal deficits when describing emotional stimuli (Gazzaniga 1988). This early evidence about the right hemisphere's dominant role in emotional speech and language was followed by subsequent patient studies in which the relative contribution of specific right hemisphere brain areas to these functions were described. Based on a large number of studies, Ross et al. concluded that the right hemisphere regulates emotional prosodic components of speech (Ross 1985; Ross and Monnot 2008) and linked the right inferior frontal cortex to expressive prosody, and the right posterior superior temporal cortex to receptive prosody. Existing clinical data confirm that right temporo-parietal lesions lead to deficits in emotional prosodic identification (Heilman et al. 1975), discrimination (Tucker et al. 1977), and recognition (Bowers et al. 1987). More recent patient data collected during intrasurgical electrocortical stimulation indicate that the right fronto-central operculum is sensitive to emotional prosody (Montavont et al. 2005). In addition to the involvement of posterior temporal areas, some patient data link right fronto-parietal (Starkstein et al. 1994), right orbito-frontal (Hornak et al. 1996), as well as right frontal brain regions (Breitenstein et al. 1998) to the recognition of emotional speech prosody.

However, not only emotional prosody but also the integration of emotional prosody with emotional semantics has been linked to right hemisphere brain regions. For instance, Lalande et al. (1992) reported that patients with right hemisphere lesions do worse than left hemisphere patients not only on emotional prosodic judgment, but also when judging the congruency of emotional prosody and semantics. Further evidence reported by Wechsler (1973) showed that right hemisphere lesion patients were less successful than left hemisphere patients in recalling emotionally laden stories when compared to neutral stories. In a series of studies, Borod et al. (1992; Cicero et al. 1999) reported impaired discrimination and identification of emotional word reading in right but not left hemisphere patients. The same group also reported stronger right than left hemisphere deficits when patients had to identify multiple emotional channels (e.g. prosodic, lexical, facial) at the same time (Borod et al. 1998a,b).

Based on these early clinical data further investigations probed whether the right hemisphere truly dominates the processing of emotional expressions. Predominantly the processing of emotional words was tested in visual hemifield studies (e.g. Brody et al. 1987; Bryden and Ley 1983; Eviatar and Zaidel 1991; Kanske and Kotz 2007; Landis 2006; Ortigue et al. 2004; Schapkin et al. 2000; Strauss 1983; Thierry and Kotz 2008) in a variety of tasks (e.g. emotional categorization, lexical decision) with supra- or subliminal presentation (Bernat et al. 2001). Interestingly, only a minority of these studies (Brody et al. 1987; Bryden and Ley 1983; Landis 2006; Ortigue et al. 2004) support a strict dominance of the right hemisphere for emotional word processing. In fact, even some early patient 
work does not confirm a strict lateralized preference for emotional speech and language processes in the right hemisphere: For instance, Blonder et al. (1991) reported no accuracy differences between controls and left hemisphere and right hemisphere patients in an emotional sentence categorization task that included sentences such as 'all the puppies are dead' spoken with a sad tone of voice (Blonder et al. 1991; Experiment 3). Furthermore, Schlanger et al. (1976) described emotional prosodic comprehension deficits in both left hemisphere and right hemisphere patients. Similarly, Cancelliere and Kertesz (1990) reported that patients with right and left hemisphere lesions of the basal ganglia display difficulties with processing emotional prosody. Finally, Van Lancker and Sidtis (1992) showed that left and right hemisphere lesion patients performed similarly in an emotion identification task in which patients had to match emotional prosodic speech samples with appropriate facial expressions/emotional labels.

However, not only patient data but also recent neuroimaging results question a strong right hemisphere lateralization of emotional speech and language function. While some data confirm an important role of right cortical and subcortical structures (e.g. right temporal and amygdala activation: Tabert et al. 2001), the majority of single word fMRI studies confirm the contribution of either hemispheres or even the left hemisphere only to emotional speech and language processing. For example, Luo et al. (2004) presented emotional words visually and subliminally and reported left mid-fusiform gyrus activation for emotional words. In addition, Strange and Dolan (2001) first reported left amygdala and hippocampus activation when emotional nouns were embedded in an oddball paradigm. In this study, emotional nouns were presented within a stream of non-emotional nouns (e.g. attic, storage, container, cabinet, warehouse, morgue, and locker, where morgue is the emotional oddball). Independent of stimulus attributes (perceptual, semantic, emotional), the authors also found activation in the right inferior prefrontal and bilateral fusiform cortices. Lewis et al. (2007) discussed a dissociation of valence in subregions of the orbito-frontal cortex and arousal in the amygdalae for visual word stimuli. In fact, left-lateralized amygdala activation in response to visually presented emotional word stimuli has been reported repeatedly. In a study by Hamann and Mao (2001), participants were asked to pay attention to visually presented words but had to make no overt response to them. Results showed increased activation in the left amygdala for both positive and negative emotional words in contrast to neutral words. In addition, positive words activated dorsal and ventral striatal regions in both hemispheres. Similarly, Herbert et al. (2009) recently reported increased activation in the left amygdala and left extrastriate visual cortex when reading pleasant as compared to unpleasant or neutral adjectives. The authors argued that the left amygdala supports salience or relevance detection during the reading of emotional words. Finally, left hemisphere involvement during visual emotional word processing was also confirmed by Kuchinke et al. (2005). Participants carried out a lexical decision task while brain activation was measured in response to emotional and neutral nouns. The authors report a predominantly left-lateralized neural network for processing emotional as opposed to neutral words (Kuchinke et al. 2005).

Acoustic presentation of emotional words also results in bilateral activation patterns. For instance, enhanced bilateral mid-superior temporal sulcus (STS) activation is reported for angry in contrast to neutral prosody (Grandjean et al. 2005). In addition, both angrily and happily intoned adjectives elicit stronger mid-STS activation irrespective of whether participants are asked to rate content or prosody valence of words (Ethofer et al. 2006). During passive listening increased activation was found in the right primary auditory cortex, the mid-superior temporal gyrus as well as the left temporal pole and hypothalamus when comparing emotional to neutral prosody during emotional word processing 
(Wiethoff et al. 2008). Moreover, auditory presentation of emotional words also elicit activation of the anterior frontal cortex (Cato et al. 2004; Crosson et al. 1999, 2002; Schirmer et al. 2004), the posterior cingulate gyrus (Maddock and Buonocore 1997; Maddock et al. 2003), and the retrosplenial cortex (Cato et al. 2004; Maddock 1999). Note, that the activation hot spots in these studies are primarily left lateralized. However, some of these results need to be viewed with caution as some only scanned the left hemisphere (Crosson et al. 1999, 2002).

Thus, combined visual and auditory clinical and neuroimaging data clearly suggest that the neural correlates of emotional speech and language span a broad network, which involves subsets of brain regions as a function of stimulus type (word, utterance) and modality, and do not strictly adhere to functional asymmetry. Also, in an attempt to account for equivocal reports on brain asymmetries, it has repeatedly been suggested that factors such as task demands, stimuli differences, experimental procedures, or participant's demography can affect the experimental results (e.g. Borod et al. 1998a,b). For example, the notion that task demands play a crucial role in emotional speech was recently confirmed by Ethofer et al. (2009a,b) who reported a network consisting of temporal cortices, the amygdalae, the insulae, and the mediodorsal thalami to respond sensitive to angry (compared to neutral) prosody irrespective of task demands. Interestingly, activation of the right mid-temporal region and the bilateral orbito-frontal cortex was influenced by increasing task demands and sensory aspects of the emotional stimuli confirming that task sensitive areas such the orbito-frontal cortex may also respond to stimulus quality.

To further explore the neural correlates of emotional speech and language, many neuroimaging studies have investigated the relative contribution of verbal and non-verbal (i.e. prosodic) expressions by pitting the two information sources against each other. The motivation of most of these studies was to achieve an inter-hemispheric (i.e. lateralization) as well as intrahemispheric specialization of the brain bases of emotional verbal and non-verbal expressions. However, a differentiation or even lateralization of verbal and non-verbal emotional expressions is hardly supported by these studies. A number of them report bilateral activation of the posterior part of the superior temporal cortex (STG; Grandjean et al. 2005; Kotz et al. 2003; Phillips et al. 1998;; but see Ethofer et al. 2009a,b; Mitchell et al. 2003; Wildgruber et al. 2002, 2004, 2005). This bilateral activation pattern implies that verbal and non-verbal emotional information naturally integrates and does not solely rely on right temporal brain regions. As discussed elsewhere (Schirmer and Kotz 2006), posterior STG activation overlaps with areas reported in voice recognition (Belin et al. 2004) suggesting that listeners are potentially drawn toward emotional tone (as realized by voice) rather than neutral tone. In addition, significant blood flow changes in the right dorsal and ventral prefrontal and orbito-frontal cortex have been reported during emotional prosodic judgment (Gandour et al. 2003; George et al. 1996; Morris et al. 1999; but see Buchanan et al. 2000; Kotz et al. 2003; Mitchell et al. 2003; Wildgruber et al. 2004, 2005 for bilateral activation). Furthermore, as indicated in patient studies, subcortical structures, such as the basal ganglia, seem to be involved in processing emotional prosody (Breitenstein et al. 1998, 2001; Paulmann and Pell 2010a; Pell and Leonard 2003; Sidtis and Van Lancker-Sidtis 2003; Wittfoth et al. 2010), emotional semantics (Castner et al. 2007; Hillier et al. 2007; Paulmann and Pell 2010a), or the integration of emotional prosody and semantics (see Paulmann and Pell 2010a for complementary electroencephalography patient data). To date, three fMRI experiments also report enhanced activation of the basal ganglia during the processing of auditory verbal (Kotz et al. 2005) and non-verbal emotion expressions (Kotz et al. 2003; Morris et al. 1999; Wildgruber et al. 2002). In addition, researchers have also advanced 
the idea that the corpus callosum (CC) and inferior frontal regions are critical when integrating information from verbal and non-verbal (prosodic) expressions (Beaucousin et al. 2007; Ethofer et al. 2006; Friederici and Alter 2004; Mitchell 2006; Mitchell et al. 2007; Schirmer et al. 2004). If the processing and the integration of different emotional information types are indeed based in a number of intra and interhemispheric brain regions, the interhemispheric transfer function of the CC becomes rather relevant for information transfer between the hemispheres.

Taken together, the current state of the art neuroimaging and clinical evidence clearly points to a diversified brain network that recruits cortical as well as subcortical brain structures in both hemispheres to process verbal and non-verbal aspects of emotional speech and language. Most authors agree that a network including temporal, fronto-parietal as well as frontal and orbito-frontal cortices, the amygdalae and the basal ganglia are recruited during emotional speech and language processing (e.g. Ethofer et al. 2009a,b; Grandjean et al. 2005; Kotz et al. 2003; Wildgruber et al. 2005). In addition, it is agreed that emotional speech perception can be divided into several subprocesses (e.g. Kotz et al. 2006; Schirmer and Kotz 2006; Wildgruber et al. 2006, 2009), though few models have tried to precisely describe the temporal dynamics of participating brain structures despite recent advancements in our understanding of the timing of processes thanks to electrophysiological data.

\section{What is the Time-Course Underlying the Emotional Speech and Language Network? Answers from Clinical and Electrophysiological Studies}

Event-related brain potentials are an excellent tool to specify the time-course of subprocesses as they measure emotional speech and language processes unfolding in time. Specifically, neuronal activity that is generated when brain cells are activated can be recorded by means of the electroencephalography. Scalp electrodes are used to record voltage fluctuation between two electrode sites in real time. An ERP component includes neural brain activity time-locked to a stimulus onset (e.g. sentence presentation) averaged over many similar stimuli (e.g. all neutral sentences). Thus, an ERP component is argued to reflect the neuronal activity that can be linked to a specific mental operation (e.g. listening to a sentence). As the electrical energy generated by brain cells is recorded in real time, ERPs have an advantage over standard behavioral measures (reaction times, accuracy scores) which measure at discrete points in time, i.e. ERPs can tap into different processing stages, including early/mandatory and late/conscious stages. In fact, several ERP components have been linked to distinct emotional speech and language processing stages; early components are argued to be influenced by physical features of a stimulus while later components are thought to reflect more cognitive based operations in response to a stimulus (e.g. Hillyard and Picton 1987). Specifically, the extraction of acoustic cues is reflected in a negativity peaking $100 \mathrm{~ms}$ after the vocal emotional stimulus onset (N100), while the integrating of these cues that impact the emotional significance of a stimulus is linked to the P200 (Paulmann and Kotz, 2008a). More in-depth 'meaning-related' evaluation of emotional vocal expressions is thought to be reflected in later negativities (N300, N400; e.g. Bostanov and Kotchoubey 2004; Paulmann and Pell 2010b; Schirmer et al. 2002, 2005; see below). Similarly, the N100-P200 complex [but see also reports on the early posterior negativity (EPN); Kissler et al. 2006] and the N300/N400 have been described in response to visual emotional stimuli supporting the notion that early and late ERP responses may not be influenced by stimulus modality. In addition, a late positive component (LPC) is often reported in visual, but also in some 
auditory studies. Altogether, latency-dependent ERP responses to the unfolding emotional stimulus confirm that ERPs are an ideal tool to investigate the complex cognitive architecture underlying emotional speech and language processing. Next to their excellent temporal resolution, ERPs have an additional advantage over behavioral methodologies as they do not require an overt response. This allows the investigation of emotional speech and language comprehension under implicit (not focussed on process of interest) and explicit (focussed on process of interest) task instructions (also see above).

For instance, ERP word level investigations report early and late emotion effects that may be modality independent (see for an overview Kissler et al. 2006). Early components such as the N100 of the N1-P2 complex are often unaffected by the emotional stimulus quality (but see Bernat et al. 2001), while the P200 is sensitive to the emotional quality of a stimulus. Both negative and positive words elicit larger P200 amplitudes than neutral words (Begleiter and Platz 1969; Begleiter et al. 1979; Bernat et al. 2001; Schapkin et al. 2000). For instance, in an early study, Begleiter and Platz (1969) presented ‘taboo' (e.g. shit, fuck) and neutral (e.g. tile, page) words to participants. They reported a differentiation between these word types within the first $200 \mathrm{~ms}$ after stimulus onset. Recently, Bernat et al. (2001) presented pleasant (affectionate) and unpleasant (agitated) words in supra- and subliminal presentation condition. The authors also report a differentiation (larger effect for unpleasant words) as early as $200 \mathrm{~ms}$. The early P200 effect for verbal expressions is generally reported to be broadly distributed (i.e. over both left and right hemisphere electrode-sites) and is thought to reflect an automatic response to emotional stimuli (but see Begleiter et al. 1979) as the effect is found in passive viewing conditions (Begleiter and Platz 1969) and in subliminal presentation (Bernat et al. 2001). However, often an enhanced P200 amplitude is linked to attentional consequences of the task (Näätänen 1992). Furthermore, some paradigms (e.g. rapid serial visual presentation) and tasks (e.g. passive viewing) also give rise to another early component that follows the N1-P2 complex, the EPN. This negative-going component occurs 200-300 ms poststimulus word onset (Kissler et al. 2006; Herbert et al. 2008; Schacht and Sommer 2009a,b; Scott et al. 2009), but also during emotional picture processing (Flaisch et al. 2008). For instance, Kissler et al. (2007) reported enhanced EPN responses to emotional compared to neutral nouns during reading at left occipito-temporal electrode-sites. Similarly, Herbert et al. (2008) described a bilaterally increased EPN for emotional compared to neutral adjectives. These findings extend reports on enhanced EPNs to emotional verbs during reading by Schacht and Sommer (2009a,b). Thus, independent of word class the reading of emotional compared to neutral words seems to modulate the EPN. Similar to the P200 reported in emotional speech, this early negativity is interpreted to reflect enhanced selective attention to emotional words leading to preferential, more in-depth processing of these words (Herbert et al. 2006). While it is assumed that the P200 may originate from the right anterior STS in the auditory domain, the EPN has been linked to extra-striate visual cortex (e.g. Junghöfer et al. 2001).

Late emotion effects for both single word verbal and non-verbal emotional expressions are reported for the P300 (Naumann et al. 1992), N400 (Kiehl et al. 1999; Williamson et al. 1991), and more frequently for the LPC (Naumann et al. 1997; Schapkin et al. 2000; Herbert et al. 2006; Dillon et al. 2006; Gibbons 2009; Schacht and Sommer (2009a,b) again dependent on experimental design (e.g. oddball) and task instructions (implicit vs. explicit emotional evaluation). These components are often distributed equally over both hemispheres. Generally speaking, emotional stimuli elicit a larger LPC amplitude than neutral stimuli and the LPC is further modulated if an emotional stimulus is highly arousing (Cuthbert et al. 2000). It is suggested that these late components reflect 
more elaborate (e.g. building-up of emotional representation and/or construction of emotional meaning) emotional processing stages (cf. Schacht and Sommer (2009a,b)).

Next to exploring when emotional stimuli first differ from neutral stimuli during single word processing, several studies have started to explore when the underlying emotional meaning of these words is first accessed. For instance, Bostanov and Kotchoubey (2004) investigated the recognition of emotional prosody using exclamations as stimuli (e.g. 'Wow', 'Oooh') in a passive oddball paradigm. They report an N300 to contextually incongruous exclamations. Also, Paulmann and Pell (2010b) recently tested the processing of emotionally intoned sentence fragments in an ERP priming paradigm. The authors report an N400-like differentiation for emotionally related prime-target pairs when compared to unrelated prime-target pairs. Similar results were reported by Schirmer and Kotz (2003) applying a word-interference paradigm (e.g. 'happy' spoken in an angry voice). Again, emotionally incongruent word pairs elicited larger N400 amplitudes than emotionally congruent word pairs. Taken together, these results confirm that emotional meaning (provided via prosody or word content) is extracted within 300-400 ms after stimulus onset. Similar to visual word stimuli, auditory stimuli very short in nature (e.g. 200 ms duration as in Paulmann and Pell 2010b) can also provide emotional meaning arguing once more for rapid recognition of emotionally relevant details.

Investigations of phrase and sentence level emotional expressions have been primarily undertaken in the auditory domain (but see Fischler and Bradley 2006 for rare visual data). Again, the N100 and P200 ERP components are involved in early emotional sentence processing. The N100 component is generally associated with the processing of sensory information such as frequency and intensity of a stimulus, and does not respond to the emotional impact of a stimulus (Pourtois et al. 2000; see also evidence for word stimuli above). The P200 is sensitive to the emotional quality of an auditory stimulus independent of the speaker voice (Paulmann and Kotz 2008a; Paulmann et al. 2008b), and reflects stimulus characteristics, such as pitch (Pantev et al. 1996), intensity (Picton et al. 1977), and arousal (Paulmann and Kotz 2006). It is also elicited with or without emotional semantic information (e.g. pseudo-sentences; Paulmann et al. 2008a). Functionally speaking, the P200 is argued to reflect initial emotional salience detection (Paulmann and Kotz 2008a), very similar to the processes linked to the visually evoked EPN; this early emotional appraisal is then followed by more elaborated stimulus evaluation. Indeed, the P200 often co-occurs with the N300, a component that has been linked to a more in-depth 'meaning-related' evaluation of a stimulus (Lebib et al. 2004; Wu and Coulson 2007) and/or a late positivity (e.g. Paulmann 2006). Similar to fMRI studies, ERP investigations also explored a potential distinction of verbal and non-verbal expressions, and show lateralized and/or distinct late ERP responses (i.e. LPC; P300) when processing semantics, prosody, or both (Grandjean et al. 2002; Kotz and Paulmann 2007; Paulmann and Kotz 2008b; Pihan 2006; Pihan et al. 1997; Schirmer and Kotz 2003; Wambacq and Jerger 2004; Wambacq et al. 2004). Such results underline the idea that verbal and non-verbal emotional expressions, such as prosody rely at least partially on distinct neural mechanisms. However, topographic indices of ERPs and DC-potentials (see Pihan 2006; Pihan et al. 1997) need to be viewed with caution as lateralized scalp surface effects may reflect a summation of generators that are not localized in one specific brain region or hemisphere. Thus, to explore which brain areas may be critically tied to the different processing steps introduced earlier (emotional salience detection, meaning-related processing, and evaluation of stimuli), we recently suggested to consider the potential of ERP lesion studies (Paulmann et al. 2010) as they allow investigating the role of a functionally critical brain area by means of a high temporal resolution methodology. 
Following this idea, we explored the role of the basal ganglia and orbito-frontal cortex in different stages of emotional speech processing (Paulmann et al. 2008a, 2009, 2010). Initial evidence suggests that early emotional salience detection, as linked to the P200, is largely unaffected by lesions to the basal ganglia (Paulmann 2006) or the orbito-frontal cortex (Paulmann et al. 2010). In contrast, the same patients showed impairment of meaning-related processing (basal ganglia patients; Paulmann et al. 2008a, 2009) and/or late evaluative-judgment and recognition related responses (both patient groups: Paulmann et al. 2008a, 2009, 2010; and see Wieser et al. 2006 for complimentary findings in the visual modality). Taken together, these latter reports help to understand which processing steps may be critically modulated by which brain structure in the emotion network. Specifically, they suggest that neither the basal ganglia nor the orbito-frontal cortex seem to be critically tied to early sensory analyses of emotional vocal stimuli nor to emotional salience detection. Rather it seems that these brain structures are particularly implied during emotional integration processes (basal ganglia) or processes that require explicit judgment of emotional stimuli such as in categorization or identification tasks (basal ganglia and orbito-frontal cortex). Clearly, these results highlight the advantage of ERP lesion studies when investigating specific processing steps.

\section{An Attempt to Integrate Findings: Modeling Emotional Speech as a Multistep Process}

Building on the evidence elaborated above, Schirmer and Kotz (2006) have argued that it is necessary to define emotional speech processing as a multistep process rather than a holistic concept. This would render the lateralization of verbal and non-verbal emotion expressions a less controversial issue. By dividing emotional prosodic processing into three subprocesses, that is, (1) acoustic analysis, (2) derivation of emotional meaning based on acoustic cues, and (3) evaluation processes, two critical aspects influencing lateralization are put forward: (1) lateralization may vary as a function of the proposed subprocesses, and (2) lateralization can be substantiated by a brain network supporting these subprocesses beyond the right hemisphere. Taking both, neuroimaging evidence with its high spatial resolution and ERP evidence with its high temporal resolution into consideration, the following was proposed (Schirmer and Kotz 2006): Acoustic analysis of temporal and frequency properties of a signal is supported by left and right primary and secondary auditory cortices, respectively (Zatorre and Belin 2001; Liegeois-Chauvel et al. 2004; Zaehle et al. 2004; but see Boemio et al. 2005 for a more fine-grained analysis of the lateralization of temporal properties of the acoustic signal). As reported in two recent fMRI studies lateralization of basic acoustic information such as temporal information may occur when integration of such information is in focus. Increased activation in left posterior auditory cortex was reported as a consequence of a perceptual switch from non-speech to speech perception requiring integration of temporal information (Dehaene-Lambertz et al. 2005; Meyer et al. 2005). When frequency or intensity information is discriminated (i.e. contrasting two tones), a more extensive right-lateralized superior temporal network including the posterior superior temporal gyrus is recruited (Liebenthal et al. 2003). Latter evidence points to the fact that the activation may not result from complex acoustic cue analysis per se, but may occur as a function of discrimination or contrast realized in an experimental set-up. The role of task demands (i.e. allocation of attention) in this context awaits further neuroimaging investigations (but see Kotz et al. 2006; Ethofer et al. 2009a,b; Wiethoff et al. 2009). However, some recent ERP evidence points to such effects (Rinne et al. 2005).

Attributing emotional significance to a stimulus when comparing emotional and neutral acoustic signals results in activations along the bilateral posterior superior temporal cortex 
overlapping with areas identified as voice-specific (Belin et al. 2004). However, the functional specification of anterior and middle portions of the superior temporal cortex related to emotional prosodic processing needs further investigations (but see Grandjean et al. 2005). Dependent on the task utilized to test emotional prosodic processing, the right frontal cortex extending into the orbito-frontal cortex (Hornak et al. 2003; Öngur et al. 2003 ) is recruited to explicitly evaluate the significance attributed to an emotional signal in the right posterior temporal cortex (see Paulmann et al. 2009 for complementary patient ERP data). Taken together, starting to specify lateralization as a function of level of processing allows to consolidate some of the controversial clinical and neuroimaging evidence reported above, even though further evidence is needed to substantiate and extend such a working model of verbal and non-verbal emotion expressions.

Based on overwhelming clinical and empirical evidence, the necessity to describe nonverbal emotional information such as prosody as a multidimensional process with bilateral and lateralized neural correlates is clearly justified. In a similar vein, the processing of verbal emotional information involves at least two processing stages but does not necessarily recruit right hemisphere areas to process emotional semantics. In fact, reported activation patterns often conform to areas generally ascribed to lexico-semantic processing in the posterior middle temporal gyrus and inferior frontal gyrus (see Friederici 2002). What needs to be addressed more clearly in future work is how verbal and non-verbal (prosodic) emotion expressions interact during online comprehension of emotional speech. One assumption is that interhemispheric flow via the CC is responsible for a fast semantic-prosodic interaction in emotional speech (see Schirmer and Kotz 2006; Friederici et al. 2007 on the interhemispheric flow impacting the syntax-prosody interface). For example, Klouda et al. (1988) reported a case study in which a lesion in the anterior portion of the CC leads to a processing deficit of affective and linguistic prosody. In comparison, the posterior portion of the CC is involved in the transfer of auditory information (Pollmann et al. 2002; Rumsey et al. 1996) and the learning of verbal skills (Nosarti et al. 2004). As lexico-semantic information processing relies on frontal and temporal brain regions, the integration of verbal and non-verbal emotional information could thus potentially occur via two callosal connections. Three recent fMRI results, while not explicitly discussing callosal connections, give evidence in support of CC involvement in emotional speech comprehension. Utilizing an emotional word Stroop task (Schirmer et al. 2004) and an emotional semantic sentence mismatch task (Mitchell 2006; Wittfoth et al. 2010) all authors report activation of the bilateral inferior frontal gyri. Furthermore, Wittfoth et al. (2010) report valence-specific mismatch effects in left fronto-temporal areas for happy intoned sentences with content expressing anger, while the left caudate/thalamus was activated when positive content was spoken in angry prosody. Latter result is confirmed by ERP patient data. Patients with left hemisphere lesions of the basal ganglia suffer from integration deficits when processing vocal anger and fear emotion expressions (Paulmann et al. 2008a). These studies clearly show that the integration of verbal and non-verbal emotion expressions as evidenced in bilateral activation of the inferior frontal gyri indicate interhemispheric information flow.

In our original model (Schirmer and Kotz 2006), we differentiated processes and neural correlates supporting vocal emotion expressions. This approach shed new light onto the lateralization of verbal and non-verbal emotion expressions and their specific brain activation patterns. Here, we extend the model by adding evidence on emotion expressions in the visual modality and highlight processing similarities in both modalities. Thus, in line with our previous proposal and similar suggestions by Kissler et al. (2006), we propose that the processing of emotion expressions involves subprocesses that can be 
ascribed to early (N100, P200, EPN) and late (N300, N400, LPC) processing stages. More specifically, we propose that visual and auditory emotional language and speech processing of words and sentences requires early sensory analysis of respective features (i.e. acoustic cues such as fundamental frequency, voice quality, loudness, or visual cues such as font, size, color, etc.) taking place approximately $100 \mathrm{~ms}$ after stimulus onset. While processing of auditory cues has been attributed to left and right primary and secondary auditory cortices (Kotz et al. 2003; Liegeois-Chauvel et al. 2004; Wildgruber et al. 2006; Zaehle et al. 2004; Zatorre and Belin 2001), the structural representation of visually presented words (i.e. an early analysis of visual form) has been linked to activation in the left fusiform gyrus (e.g. Dehaene et al. 2002). Once word form properties have been accessed, emotional salience is detected and emotional meaning can be determined. Within the auditory modality processing of emotional significance has been attributed to activation of the right anterior STS/STG (see Schirmer and Kotz 2006), while visually presented emotional words engage the left mid fusiform gyrus (e.g. Luo et al. 2004) and/or the left amygdala and the hippocampus as well as the left extra-striate visual cortex (Junghöfer et al. 2001), and bilateral fusiform cortices (Strange and Dolan 2001). As latter activation was reported when emotional nouns were embedded in an oddball paradigm, it can be hypothesized that these brain regions are particularly implied during emotional salience detection and early access of word meaning. In particular, some authors have stressed the role of the left amygdala during salience or relevance detection (e.g. Herbert et al. 2009). Finally, later stages of processing including integration of different channels (e.g. semantics and prosody) and information types (broader context) as well as more evaluative processes of stimuli start taking place around $400 \mathrm{~ms}$ after stimulus onset and are likely to be supported by structures such as the bilateral inferior frontal and orbito-frontal cortex. Here, the evidence points to possible amodal activation patterns (see Figure 1).

In conclusion, we propose that emotional speech and language processing includes at least three processing steps: (1) analysis of acoustic and visual features of a given stimulus, (2) derivation of emotional meaning/salience based on these sensory cues, and (3) amodal evaluation. This extension from auditory to auditory and visual processing of emotion expressions is consistent with findings from imaging studies that confirm the linking of specific ERP components to different processing stages subserved by different brain areas. Thus, a differentiated processing view of emotion expressions allows to newly evaluating diverse and controversial lesion and neuroimaging. We believe that future studies combining functional activation, white matter tracking, and functional connectivity analyses will further illuminate intra and interhemispheric neural circuitry regulating the processing of emotion.

\section{Short Biographies}

Sonja A. Kotz is a cognitive neuroscientist who investigates speech, language, emotion, rhythm and timing in healthy, clinical (aphasic, neurodegenerative), and bilingual populations using neuroimaging methods such as ERPs, MEG, AMRI, and TMS. She recently received a 5-year award from the Max Planck Society's 'W 2 Special Programme for the Advancement of Outstanding Female Scientists at the Max Planck Society' that supports her independent research group: 'Neurocognition of Rhythm in Communication' at the Max Planck Institute for Human Cognitive and Brain Sciences in Leipzig, Germany.

Silke Paulmann is a lecturer in the Department of Psychology at the University of Essex, UK. She works on emotion and language processing in normal (young, old) and 


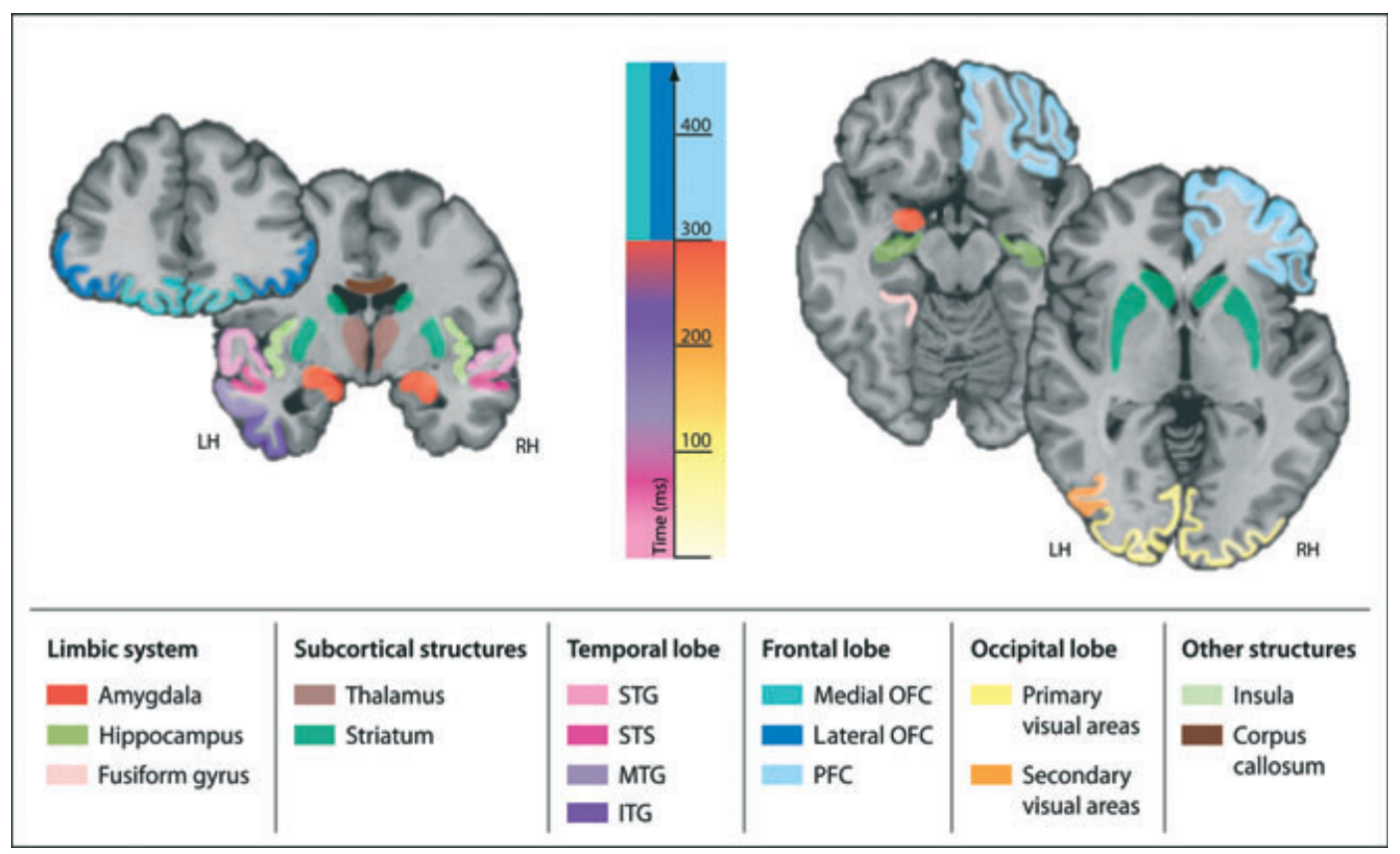

Fig 1. Displayed are in the left panel, in coronal view, activation patterns reported in auditory emotion studies and in the right panel, in axial view, the respective activation patterns in the visual modality. The time axis in the middle displays from bottom to top in colors corresponding to respective brain activations the possible time-line correspondence of ERP effects. The color coding respects common activation in both modalities where possible (e.g. primary and secondary sensory areas, subcortical structures, and frontal structures). STG, superior temporal gyrus; STS, superior temporal sulcus; MTG, middle temporal gyrus; ITG, inferior temporal gyrus; OFC, orbito-frontal cortex; PFC, prefrontal gyrus.

special populations (bilinguals, brain damaged) and investigates how the brain manages to successfully process language within milliseconds. To explore this capability her research employs event-related brain potentials (ERPs), but also behavioral methodologies and eye tracking. In the past years, she has held fellowships from the German Academic Exchange Service (DAAD) and her most recent research is supported by grants from the Centre for Research on Language, Mind and Brain (CRLMB), and the British Academy.

\section{Acknowledgement}

We gratefully acknowledge the German Research Foundation (FOR-499) and the Canadian Institutes of Health Research for ongoing financial support of this research. We also thank Andrea Gast-Sandmann for graphics support and Anika Stockert for neuroanatomical specifications.

\section{Notes}

* Correspondence address: Sonja A. Kotz, MPI for Human Cognitive and Brain Sciences, Neurocognition of Rhythm in Communication Group, Stephanstrasse 1a, Leipzig 04103, Germany. E-mail: kotz@cbs.mpg.de

1 Other non-verbal expressions include facial, gestural, or postural information sources. However, the present paper limits its review to findings from emotional speech and language studies. 


\section{Works Cited}

Adolphs, R., H. Damasio, and D. Tranel. 2002. Neural systems for recognition of emotional prosody: a 3-D lesion study. Emotion 2(1). 23-51.

$\longrightarrow,-\longrightarrow$, and A. R. Damasio. 1996. Cortical systems for the recognition of emotion in facial expressions. Journal of Neuroscience 16(23). 7678-87.

Beaucousin, V., A. Lacheret, M. R. Turbelin, M. Morel, B. Mazoyer, and N. Tzourio-Mazoyer. 2007. FMRI study of emotional speech comprehension. Cerebral Cortex 17(2). 339-52.

Begleiter, H., and A. Platz. 1969. Cortical evoked potentials to semantic stimuli. Psychophysiology 6(1). 91-100.

$\longrightarrow$, B. Projesz, and R. Garazzo. 1979. Evoked brain potentials and behavior. Visual evoked potentials and affective ratings of semantic stimuli, ed. by H. Begleiter, 127-43. New York: Plenum Press.

Belin, P., S. Fecteau, and C. Bedard. 2004. Thinking the voice: neural correlates of voice perception. Trends in Cognitive Neuroscience 8(3). 129-35.

Bernat, E., S. Bunce, and H. Shevrin. 2001. Event-related brain potentials differentiate positive and negative mood adjectives during both supraliminal and subliminal visual processing. International Journal of Psychophysiology 42(1). 11-34.

Blonder, L. X., D. Bowers, and K. M. Heilman. 1991. The role of the right hemisphere in emotional communication. Brain 114. 1115-27.

Boemio, A., S. Fromm, A. Braun, and D. Poeppel. 2005. Hierarchical and asymmetric temporal sensitivity in human auditory cortices. Nature Neuroscience 8(3). 389-95.

Borod, J. C., F. Andelman, L. K. Obler, J. R. Tweedy, and J. Welkowitz. 1992. Right hemisphere specialization for the identification of emotional words and sentences: evidence from stroke patients. Neuropsychologia 30(9). 827-44.

— R. L. Bloom, and C. Santschi-Haywood. 1998a. Verbal aspects of emotional communication. Right hemisphere language comprehension: perspectives from cognitive neuroscience, ed. by M. Beeman and C. Chiarello, 285-307. Hillsdale, NJ: Lawrence Erlbaum Associates.

—, B. A. Cicero, L. K. Obler, J. Welkowitz, H. M. Erhan, C. Santschi, I. S Grunwald, R. M. Agosti, and J. R. Whalen. 1998b. Right hemisphere emotional perception: evidence across multiple channels. Neuropsychology 12(3). 446-58.

—, K. D. Rorie, L. H. Pick, R. L. Bloom, F. Andelman, A. L. Campell, L. K. Obler, J. R. Tweedy, J. Welkowitz, and M. Sliwinski. 2000. Verbal pragmatics following unilateral stroke: emotional content and valence. Neuropsychology 14(1). 112-24.

Bostanov, V., and B. Kotchoubey. 2004. Recognition of affective prosody: continuous wavelet measures of eventrelated brain potentials to emotional exclamations. Psychophysiology 41(2). 259-68.

Bowers, D., H. Coslett, R. Bauer, L. Speedie, and K. Heilman. 1987. Comprehension of emotional prosody following unilateral hemispheric lesions: processing defect versus distraction defect. Neuropsychologia 25(2). 317-28.

Breitenstein, C., I. Daum, and H. Ackermann. 1998. Emotional processing following cortical and subcortical brain damage: contribution of the fronto-striatal circuitry. Behavioral Neurology 11(1). 29-42.

— - D. V. Lancker, I. Daum, and C. H. Waters. 2001. Impaired perception of vocal emotions in Parkinson's disease: influence of speech time processing and executive functioning. Brain and Cognition 45(2). 277-314.

Brody, N., S. Goodman, E. Halm, S. Krinzman, and M. Sebrechts. 1987. Lateralized affective priming of lateralized affectively valued target words. Neuropsychologia 25(6). 935-46.

Bryden, M., and R. Ley. 1983. Right-hemispheric involvement in the perception and expression of emotion in normal humans. Neuropsychology of human emotion, ed. by K. Heilman and P. Satz, 6-44. New York: Guildford.

Buchanan, T. W., K. Lutz, S. Mirzazade, K. Specht, N. J. Shah, K. Zilles et al. 2000. Recognition of emotional prosody and verbal components of spoken language: an fMRI study. Cognitive Brain Research 9(3). 227-38.

Cancelliere, A. E. B., and A. Kertesz. 1990. Lesion localization in acquired deficits of emotional expression and comprehension. Brain and Cognition 13(2). 133-47.

Castner, J. E., H. J Chenery, D. A. Copland, T. J. Coyne, F. Sinclair, and P. A. Silburn. 2007. Semantic and affective priming as a function of stimulation of the subthalamic nucleus in Parkinson's disease. Brain 130. $1395-407$.

Cato, M. A., B. Crosson, D. Gökçay, D. Soltysik, C. Wierenga, K. Gopinath, N. Himes, H. Belanger, R. M. Bauer, I. S. Fischler, L. Gonzalez-Rothi, and R. W. Briggs. 2004. Processing words with emotional connotation: an FRMI study of the time course and laterality in rostral frontal and retrosplenial cortices. Journal of Cognitive Neuroscience 16(2). 167-77.

Cicero, B. A., J. C. Borod, C. Santschi, H. M. Erhan, L. K. Obler, R. M. Agosti, J. Welkowitz, and I. S. Grunwald. 1999. Emotional versus nonemotional lexical perception in patients with right and left brain damage. Neuropsychiatry, Neuropsychology, and Behavioral Neurology 12(4). 255-64.

Crosson, B., M. A. Cato, J. R. Sadek, D. Gökçay, R. M. Bauer, I. S. Fischler, L. Maron, K. Gopinath, E. J. Auerbach, S. R. Browd, and R. W. Briggs. 2002. Semantic monitoring of words with emotional connotation during 
fMRI: contribution of the left anterior frontal cortex. Journal of the International Neuropsychological Society 8(5). 607-22.

—, K. Radonovich, J. R. Sadek, D. Gokcay, R. M. Bauer et al. 1999. Left-hemisphere processing of emotional connotation during word generation. NeuroReport 10(2). 2449-55.

Cuthbert, B. N., H. T. Schupp, M. M. Bradley, N. Birbaumer, and P. J. Lang. 2000. Brain potentials in affective picture processing: covariation with autonomic arousal and affective report. Biological Psychology 52(2). 95-111.

Davidson, R. J. 2000. Affective style, psychopathology, and resilience: brain mechanisms and plasticity. American Psychologist 55(11). 1196-214.

Dehaene, S., H. G. Le Clec', J-B Poline, D. Le Bihan, and L. Cohen. 2000. The visual word form area: a prelexical representation of visual words in the fusiform gyrus. NeuroReport 13. 321-5.

Dehaene-Lambertz, G., C. Pallier, W. Serniclaes, L. Sprenger-Charolles, A. Jobert, and S. Dehaene. 2005. Neural correlates of switching from auditory to speech perception. NeuroImage 24(1). 21-33.

Dillon, D. G., J. J. Cooper, T. Grent-'t-Jong, M. G. Woldorff, and K. S. LaBar. 2006. Dissociation of event-related potentials indexing arousal and semantic cohesion during emotional word encoding. Brain and Cognition 62(1). 43-57.

Ethofer, T., S. Anders, M. Erb, C. Herbert, S. Wiethoff, J. Kissler, W. Grodd, and D. Wildgruber. 2006. Cerebral pathways in processing of affective prosody: a dynamic causal modeling study. NeuroImage 30(2). 580-7.

- D. V. De Ville, K. Scherer, and P. Veuilleumeur. 2009a. Decoding of emotional information in voice-sensitive cortices. Current Biology 19(12). 1028-33.

— B. Kreifelts, S. Wiethoff, J. Wolf, W. Grodd, P. Vuilleumier, and D. Wildgruber. 2009b. Differential influences of emotion, task, and novelty on brain regions underlying the processing of speech melody. Journal of Cognitive Neuroscience 21(7). 1255-68.

Eviatar, Z., and E. Zaidel. 1991. The effects of word length and emotionality on hemispheric contributions to lexical decision. Neuropsychologia 29(5). 415-28.

Fischler, I., and M. Bradley. 2006. Event-related potential studies of language and emotion: words, phrases, and task effects. Progress in Brain Research 156. 185-203.

Flaisch, T., J. Stockburger, and H. T. Schupp. 2008. Affective prime and target picture processing: an ERP analysis of early and late interference effects. Brain Topography 20(4). 183-91.

Friederici, A. D. 2002. Towards a neural basis of auditory sentence processing. Trends in Cognitive Sciences 6(2). 78-84.

— Language 89(2). 267-76.

- D. Y. von Cramon, and S. A. Kotz. 2007. Role of the corpus callosum in speech comprehension: interfacing syntax and prosody. Neuron 53(1). 135-45.

Gandour, J., D. Wong, M. Dzemidzic, M. Lowe, Y. Tong, and X. Li. 2003. A cross-linguistic fMRI study of perception of intonation and emotion in Chinese. Human Brain Mapping 18(3). 149-57.

Gazzaniga, M. S. 1988. Brain modularity: towards a philosophy of conscious experience. Consciousness in contemporary science, ed. by A. J. Marcel and E. Bisiach, 218-38. Oxford: Oxford University Press.

George, M. S., P. I. Parekh, N. Rosinsky, T. A. Ketter, T. Kimbrell, K. M. Heilman et al. 1996. Understanding emotional prosody activates right hemisphere regions. Archives of Neurology 53(7). 665-70.

Gibbons, H. 2009. Evaluative priming from subliminal emotional words: insights from event-related potentials and individual differences related to anxiety. Consciousness and Cognition 18(2). 383-400.

Grandjean, D., C. Ducommun, P.-J. Bernard, and K. R. Scherer. 2002. Comparison of cerebral activation patterns in identifying affective prosody, semantic prosody, and phoneme differences. Poster presented at the International Organization of Psychophysiology (IOP), August, Montreal, Canada.

— , D. Sander, G. Pourtois, S. Schwartz, M. L. Seghier, K. R. Scherer, and P. Vuilleumier. 2005. The voices of wrath: brain responses to angry prosody in meaningless speech. Nature Neuroscience 8(2). 145-6.

Hamann, S., and H. Mao. 2001. FMRI correlates of emotional memory and reactions to verbal and nonverbal emotional stimuli. NeuroImage 13(6). S415.

Harciarek, M., K. M. Heilmann, and K. Jodzio. 2006. Defective comprehension of emotional faces and prosody as a result of right hemisphere stroke: modality versus emotion-type specificity. Journal of the International Neuropsychological Society 12(6). 774-81.

Heilman, K. M., R. Scholes, and R. T. Watson. 1975. Auditory affective agnosia - disturbed comprehension of affective speech. Journal of Neurology, Neurosurgery and Psychiatry 38(1). 69-72.

Herbert, C., T. Ethofer, S. Anders, M. Junghofer, D. Wildgruber, W. Grodd, and J. Kissler. 2009. Amygdala activation during reading of emotional adjectives - an advantage for pleasant content. Social, Cognitive, and Affective Neuroscience 4(1). 35-49.

— - M. Junghöfer, and J. Kissler. 2008. Event related potentials to emotional adjectives during reading. Psychophysiology 45. 487-98.

—, J. Kissler, M. Junghöfer, P. Peyk, and B. Rockstroh. 2006. Processing of emotional adjectives: evidence from startle EMG and ERPs. Psychophysiology 43(2). 197-206. 
Hillier, A., D. Q. Beversdorf, A. M. Raymer, D. J. G. Williamson, and K. M. Heilman. 2007. Abnormal emotional word ratings in Parkinson's disease. Neurocase 13(2). 81-5.

Hillyard, S. A., and T. W. Picton. 1987. Electrophysiology of cognition. Handbook of physiology: the nervous system. Vol. V, part 2, higher functions of the brain, Vol. V, ed. by F. Plum, 519-84. New York: American Physiological Society.

Hornak, J., J. Bramham, E. T. Rolls, R. G. Morris, J. O’Doherty, P. R. Bullock, and C. E. Polkey. 2003. Changes in emotion after circumscribed surgical lesions of the orbito-frontal and cingulate cortices. Brain 126. $1691-712$.

— E. T. Rolls, and D. Wade. 1996. Face and voice expression identification in patients with emotional and behavioural changes following ventral frontal lobe damage. Neuropsychologia 34(4). 247-61.

Jackson, J. H. 1874. On the nature of the duality of the brain. Selected writings of John Hughlings Jackson, ed. by J. Taylor, 804-5. Bd. 2. London: Hodder \& Stoughton.

Junghöfer, M., M. M. Bradley, T. R. Elbert, and P. J. Lang. 2001. Fleeting images: a new look at early emotion discrimination. Psychophysiology 38(2). 175-8.

Kanske, P., and S. A. Kotz. 2007. Concreteness in emotional words: ERP evidence from a hemifield study. Brain Research 1148. 138-48.

Kiehl, K. A., P. F. Liddle, A. M. Smith, A. Mendrek, B. B. Forster, and R. D. Hare. 1999. Neural pathways involved in the processing of concrete and abstract words. Human Brain Mapping 7(4). 225-33.

Kissler, J., R. Assadollahi, and C. Herbert. 2006. Emotional and semantic networks in visual word processing: insights from ERP studies. Progress in brain research, Vol. 156, ed. by S. Anders, G. Ende, M. Junghöfer, J. Kissler and D. Wildgruber, 147-83. Amsterdam: Elsevier.

—, C. Herbert, P. Peyk, and M. Junghöfer. 2007. Buzzwords-early cortical responses to emotional words during reading. Psychological Science 18. 475-80.

Klouda, G. V., D. A. Robin, N. R. Gaffradford, and W. E. Cooper. 1988. The role of callosal connections in speech prosody. Brain and Language 35(1). 154-71.

Kotz, S. A., M. Meyer, K. Alter, M. Besson, D. Y. von Cramon, and A. D. Friederici. 2003. On the lateralization of emotional prosody: an event-related functional MR investigation. Brain and Language 86(3). 366-76.

- - - a and S. Paulmann. 2006. Lateralization of emotional prosody in the brain: an overview and synopsis on the impact of study design. Progress in brain research, ed. by S. Anders, G. Ende, M. Junghöfer, J. Kissler and D. Wildgruber, 156. 285-94. Amsterdam: Elsevier.

- and S. Paulmann. 2007. When emotional prosody and semantics dance cheek to cheek: ERP evidence. Brain Research 1151. 107-18.

- , - and T. Raettig. 2005. Varying task demands during the perception of emotional content: efMRI evidence. Journal of Cognitive Neuroscience Supplement 63.

Kreifelts, B., T. Ethofer, W. Grodd, M. Erb, and D. Wildgruber. 2007. Audiovisual integration of emotional signals in voice and face: an event-related fMRI study. NeuroImage 37(4). 1445-56.

- - — T. Shiozawa, W. Grodd, and D. Wildgruber. 2009. Cerebral representation of non-verbal emotional perception: fMRI reveals audiovisual integration area between voice- and face-sensitive regions in the superior temporal sulcus. Neuropsychologia 47(14). 3059-66.

Kuchinke, L., A. M. Jacobs, C. Grubich, M. L.-H. Võ, M. Conrad, and M. Herrmann. 2005. Incidental effects of emotional valence in single word processing: an fMRI study. NeuroImage 28(4). 1022-32.

Lalande, S., C. M. Braun, N. Charlebois, and H. A. Whitaker. 1992. Effects of right and left hemisphere cerebrovascular lesions on discrimination of prosodic and semantic aspects of affect in sentences. Brain and Language 42(2). 165-86.

Landis, T. 2006. Emotional words: what's so different from just words? Cortex 42(6). 823-30.

Lebib, R., D. Papo, A. Douiri, S. de Bode, M. Gillon Dowens, and P. M. Baudonniere. 2004. Modulations of late event-related brain potentials in human by dynamic audiovisual speech stimuli. Neuroscience Letters 372(1-2). 74-9.

Lewis, P. A., H. D. Critchley, P. Rothstein, and R. J. Dolan. 2007. Neural correlates of processing valence and arousal in affective words. Cerebral Cortex 17(3). 742-8.

Liebenthal, E., R. Binder, R. L. Piorkowski, and R. E. Remez. 2003. Short-term reorganization of auditory analysis induced by phonetic experience. Journal of Cognitive Neuroscience 15(4). 549-58.

Liegeois-Chauvel, C., C. Lorenzi, A. Trebuchon, J. Regis, and P. Chauvel. 2004. Temporal envelope processing in the human left and right auditory cortices. Cerebral Cortex 14(7). 731-40.

Luo, Q., D. Peng, Z. Jin, D. Xu, L. Xiao, and G. Ding. 2004. Emotional valence of words modulates the subliminal repetition priming effect in the left fusiform gyrus: an event-related fMRI study. NeuroImage 21(3). 414-21.

Maddock, R. J. 1999. The retrosplenial cortex and emotion: new insights from functional neuroimaging of the human brain. [Review]. Trends in Neurosciences 22(7). 310-6.

- and M. H. Buonocore. 1997. Activation of left posterior cingulate gyrus by the auditory presentation of threat-related words: an fMRI study. Psychiatry Research 75(1). 1-14.

— A. S. Garrett, and M. H. Buonocore. 2003. Posterior cingulate cortex activation by emotional words: fMRI evidence from a valence decision task. Human Brain Mapping 18(1). 30-41. 
Meyer, M., T. Zaehle, V. E. Gountouna, A. Barron, L. Jaencke, and A. Turk. 2005. Spectrotemporal processing during speech perception involves left posterior auditory cortex. NeuroReport 16(18). 1985-9.

Mitchell, D. G., M. Nakic, D. Fridberg, N. Kamel, D. S. Pine, and R. J. Blair. 2007. The impact of processing load on emotion. NeuroImage 34(3). 1299-309.

Mitchell, R. 2006. Does incongruent lexico-semantic and prosodic information cause discernable cognitive conflict? Cognitive, Affective, and Behavioral Neuroscience 6(4). 298-305.

— , R. Elliott, M. Barry, A. Cruttenden, and P. W. R. Woodruff. 2003. The neural response to emotional prosody, as revealed by functional magnetic resonance imaging. Neuropsychologia 41(10). 1410-21.

Montavont, A., G. Demarquay, M. Guenot, J. Isnard, F. Mauguiere, and P. Ryvlin. 2005. Ictal dysprosody and the role of the non-dominant frontal operculum. Epileptic Disorder 7. 193-7.

Morris, J. S., S. K. Scott, and R. J. Dolan. 1999. Saying it with feeling: neural responses to emotional vocalizations. Neuropsychologia 37(10). 1155-63.

Näätänen, R. 1992. Attention and brain function. Hillsdale, NJ: Erlbaum.

Naumann, E., D. Bartussek, O. Diedrich, and M. E. Laufer. 1992. Assessing cognitive and affective information processing functions of the brain by means of the late positive complex of the event related potential. Journal of Psychophysiology 6(4). 285-98.

— , S. Maier, O. Diedrich, G. Becker, and D. Bartussek. 1997. Structural, semantic, and emotion-focused processing of neutral and negative nouns: event-related potential correlates. Journal of Psychophysiology 11(2). 15872.

Nosarti, C., T. M. Rushe, P. W. R. Woodruff, A. L. Stewart, L. Rifkin, and R. M. Murray. 2004. Corpus callosum size and very preterm birth: relationship to neuropsychological outcome. Brain 127. 2080-9.

Öngur, D., A. T. Ferry, and J. L. Price. 2003. Architectonic subdivision of the human orbital and medial prefrontal cortex. The Journal of Comparative Neurology 460. 425-49.

Ortigue, S., C. M. Michel, M. M. Murray, C. Mohr, S. Carbonnel, and T. Landis. 2004. Electrical neuroimaging reveals early generator modulation to emotional words. NeuroImage 21(4). 1242-51.

Pantev, C., T. Elbert, B. Ross, C. Eulitz, and E. Terhardt. 1996. Binaural fusion and the representation of virtual pitch in the human auditory cortex. Hearing Research 100. 164-70.

Paulmann, S. 2006. Electrophysiological evidence on the processing of emotional prosody: insights from healthy and patient populations. MPI series in human cognitive and brain sciences, Vol. 71, p. 281. Leipzig: Max Planck Institute for Human Cognitive and Brain Sciences.

- and S. A. Kotz. 2006. Valence, arousal, and task effects on the P200 in emotional prosody processing. In the Proceedings of Architectures and Mechanisms for Language Processing 2006 (AMLaP 2006), Nijmegen, the Netherlands, p. 37.

— , and 2008a. Early emotional prosody perception based on different speaker voices. NeuroReport 19(2). 209-13.

— , and 2008b. An ERP Investigation on the temporal dynamics of emotional prosody and emotional semantics in pseudo- and lexical sentence context. Brain and Language 105(1). 59-69.

— , and M. D. Pell. 2010a. Dynamic emotion processing in Parkinson's disease as a function of channel availability. Journal of Clinical and Experimental Neuropsychology 32(8). 822-35.

— , and 2010b. Contextual influences of emotional speech prosody on face processing: how much is enough? Cognitive, Affective, and Behavioral Neuroscience 10. 230-42.

,-- , and S. A. Kotz. 2008a. Functional contributions of the basal ganglia to emotional prosody: evidence from ERPs. Brain Research 1217. 171-8.

,-- , and - 2009. Comparative processing of emotional prosody and semantics following basal ganglia infarcts: ERP evidence of selective impairments for disgust and fear. Brain Research 1295. 159-69.

- , P. Schmidt, M. D. Pell, and S. A. Kotz. 2008b. Rapid processing of emotional and voice information as evidenced by ERPs, SP-2008, 205-9.

- S. Seifert, and S. A. Kotz. 2010. Orbito-frontal lesions cause impairment in late but not early emotional prosodic processing stages. Social Neuroscience 5. 59-75.

Pell, M. D., and C. L. Leonard. 2003. Processing emotional tone from speech in Parkinson's disease: a role for the basal ganglia. Cognitive, Affective, and Behavioral Neuroscience 3(4). 275-88.

Phillips, M. L., A. W. Young, S. K. Scott, A. J. Calder, C. Andrew, V. Giampietro et al. 1998. Neural responses to facial and vocal expressions of fear and disgust. Proceedings of the Royal Society of London Series B, Biological Sciences 265. 1809-17.

Picton, T. W., D. L. Woods, J. Baribeau-Braun, and T. M. G. Healey. 1977. Evoked potential audiometry. Journal of Otolaryngology 6(2). 90-119.

Pihan, H. 2006. Affective and linguistic processing of speech prosody: DC potential studies. Progress in Brain Research 156. 269-84.

— E. Altenmüller, and H. Ackermann. 1997. The cortical processing of perceived emotion: a DC-potential study on affective speech prosody. NeuroReport 8(3). 623-7. 
Pollmann, S., M. Maertens, D. Y. von Cramon, J. Lepsien, and K. Hugdahl. 2002. Dichotic listening in patients with splenial and nonsplenial callosal lesions. Neuropsychology 16(1). 56-64.

Pourtois, G., B. de Gelder, J. Vroomen, B. Rossion, and M. Crommelinck. 2000. The time-course of intermodal binding between seeing and hearing affective information. NeuroReport 11(6). 1329-33.

Rinne, T., J. Pekkola, A. Degerman, T. Autti, I. P. Jaaskelainen, M. Sams, and K. Alho. 2005. Modulation of auditory cortex activation by sound presentation rate and attention. Human Brain Mapping 26(2). 94-9.

Ross, E. D. 1985. Modulation of affect and nonverbal communication by the right hemisphere. Principles of behavioral neurology, ed. by M. M. Mesulam, 239-58. Philadelphia, PA: Davis.

—, and M. Monnot. 2008. Neurology of affective prosody and its functional-anatomic organization in right hemisphere. Brain and Language 104(1). 51-74.

Rumsey, J. M., M. Casanova, G. B. Mannheim, N. Patronas, N. DeVaughn, S. D. Hamburger, and T. Acquino. 1996. Corpus Callosum morphology, as measured with MRI, in dyslexic men. Biological Psychiatry 39. 769-75.

Schacht, A., and W. Sommer. 2009a. Time course and task dependence of emotion effects in word processing. Cognitive, Affective, and Behavioral Neuroscience 9. 28-43.

— , and 2009b. Emotions in word and face processing: early and late cortical responses. Brain and Cognition 69. 538-50.

Schapkin, S. A., A. N. Gusev, and J. Kuhl. 2000. Categorization of unilaterally presented emotional words: an ERP analysis. Acta Neurobiolgiae Experimentals 60(1). 17-28.

Schirmer, A., and S. A. Kotz. 2003. ERP evidence for a sex-specific Stroop effect in emotional speech. Journal of Cognitive Neuroscience 15(8). 1135-48.

— , and - 2006. Beyond the right hemisphere: brain mechanisms mediating vocal emotional processing. Trends in Cognitive Sciences 10(1). 24-30.

- - - , and A. D. Friederici. 2002. Sex differentiates the role of emotional prosody during word processing. Cognitive Brain Research 14. 228-33.

- - - , and - 2005. On the role of attention for the processing of emotions in speech: sex differences revisited. Cognitive Brain Research 24. 442-52.

— S. Zysset, S. A. Kotz, and D. Y. von Cramon. 2004. Gender differences in the activation of inferior frontal cortex during emotional speech perception. NeuroImage 21(3). 1114-23.

Schlanger, B. B., P. Schlanger, and L. J. Gerstmann. 1976. The perception of emotionally toned sentences by right hemisphere-damaged and aphasic subjects. Brain and Language 3. 396-403.

Scott, S. K., and I. S. Johnsrude. 2003. The neuroanatomical and functional organization of speech perception. Trends in Neurosciences 26. 100-7.

Scott, G. G., P. J. O’Donnell, H. Leuthold, and S. C. Sereno. 2009. Early emotion word processing: evidence from event-related potentials. Biological Psychology 80. 95-104.

Sidtis, J. J., and D. Van Lancker-Sidtis. 2003. A neurobehavioral approach to dysprosody. Seminars in Speech Language 24. 93-105.

Smith, A. 1966. Speech and other functions after left (dominant) hemispherectomy. Journal of Neurology, Neurosurgery, and Psychiatry 29. 467-71.

Starkstein, S. E., J. P. Federoff, T. R. Price, R. C. Leiguarda, and R. G. Robinson. 1994. Neuropsychological and neuroradiologic correlates of emotional prosody comprehension. Neurology 44(3 Pt 1). 515-22.

Strange, B. A., and R. J. Dolan. 2001. Adaptive anterior hippocampal responses to oddball stimuli. Hippocampus 11(6). 690-8.

Strauss, E. 1983. Perception of emotional words. Neuropsychologia 21(1). 99-103.

Tabert, M. H., J. C. Borod, C. Y. Tang, G. Lange, T. C. Wei, R. Johnson, A. O. Nusbaum, and M. C. Buchsbaum. 2001. Differential amygdala activation during emotional decision and recognition memory tasks using unpleasant words: an fMRI study. Neuropsychologia 39(6). 556-73.

Thierry, G., and S. A. Kotz. 2008. The right hemisphere fails to orient to the negative valence of visually presented words. NeuroReport 19(12). 1231-4.

Tucker, D. M., R. T. Watson, and K. M. Heilman. 1977. Discrimination and evocation of affectively intoned speech in patients with right parietal disease. Neurology 27(10). 947-50.

Van Lancker, D., and J. J. Sidtis. 1992. The identification of affective-prosodic stimuli by left- and right-hemisphere-damaged subjects: all errors are not created equal. Journal of Speech and Hearing Research 35. 936-70.

Wambacq, I. J. A., and J. F. Jerger. 2004. Processing of affective prosody and lexical-semantics in spoken utterances as differentiated by event-related potentials. Cognitive Brain Research 20. 427-37.

- , K. J. Shea-Miller, and A. Abubakr. 2004. Non-voluntary and voluntary processing of emotional prosody: an event-related potentials study. NeuroReport 15(3). 555-9.

Wechsler, A. F. 1973. The effect of organic brain disease on recall of emotionally charged versus neutral narrative texts. Neurology 23(2). 130-5.

Wieser, M., A. Mühlberger, G. W. Alpers, M. Macht, H. Ellgring, and P. Pauli. 2006. Emotion processing in Parkinson's disease: dissociation between early neuronal processing and explicit ratings. Clinical Neurophysiology 117(1). 94-102. 
Wiethoff, S., D. Wildgruber, H. Becker, S. Anders, C. Herbert, W. Grodd, and T. Ethofer. 2008. Cerebral processing of emotional prosody - influence of acoustic parameters and arousal. NeuroImage 39. 885-93.

- - W W. Grodd, and T. Ethofer. 2009. Response and habituation of the amygdala during processing of emotional prosody. NeuroReport 20(15). 1356-60.

Wildgruber, D., H. Ackermann, B. Kreifelts, and T. Ethofer. 2006. Processing of linguistic and emotional prosody: fMRI studies. Progress in Brain Research 156. 249-68.

, T. Ethofer, D. Grandjean, and B. Kreifelts. 2009. A cerebral network model of speech prosody comprehension. International Journal of Speech-Language Pathology 11. 277-81.

- I. Hertrich, A. Riecker, M. Erb, S. Anders, W. Grodd et al. 2004. Distinct frontal regions subserve evaluation of linguistic and emotional aspects of speech intonation. Cerebral Cortex 14(12). 1384-9.

- - H. Pihan, H. Ackermann, M. Erb, and W. Grodd. 2002. Dynamic brain activation during processing of emotional intonation: influence of acoustic parameters, emotional valence, and sex. NeuroImage 15(4). 856-69.

—, A. Riecker, I. Hertrich, M. Erb, W. Grodd, T. Ethofer, and H. Ackermann. 2005. Identification of emotional intonation evaluated by fMRI. NeuroImage 24(4). 1233-41.

Williamson, S., T. J. Harpur, and R. D. Hare. 1991. Abnormal processing of affective words by psychopaths. Psychophysiology 28(3). 260-73.

Wittfoth, M., C. Schroder, D. M. Schardt, R. Dengler, H. J. Heinze, and S. A. Kotz. 2010. On emotional conflict: interference resolution of happy and angry prosody reveals valence-specific effects. Cerebral Cortex 20(2). 38392.

Wu, Y. C., and S. Coulson. 2007. How iconic gestures enhance communication: an ERP study. Brain and Language 101(3). 234-45.

Zaehle, T., T. Wustenberg, M. Meyer, and L. Jaencke. 2004. Evidence for rapid auditory perception as the foundation of speech processing: a sparse temporal sampling fMRI study. European Journal of Neuroscience 20. 244756.

Zatorre, R. J., and P. Belin. 2001. Spectral and temporal processing in human auditory cortex. Cerebral Cortex 11(10). 946-53.

Zupan, B., D. Neumann, D. R. Babbage, and B. Willer. 2009. The importance of vocal affect to bimodal processing of emotion: implications for individuals with traumatic brain injury. Journal of Communication Disorders 42(1). 1-17. 